\title{
Four variations in global Carleman weights applied to inverse and controllability problems*
}

\author{
AXEL OSSES
}

Departamento de Ingenería Matemática, Facultad de Ciencias de Físicas y Matemáticas Universidad de Chile, Casilla 170/3, Correo 3, Santiago, Chile y Centro de Modelamiento Matemático, UMI 2807 CNRS-Uchile

E-mail: axosses@dim.uchile.cl

\begin{abstract}
This paper reviews four variants of global Carleman weights that are especially adapted to some singular controllability and inverse problems in partial differential equations. These variants arise when studying: i) one measurement stationary source inverse problems for the heat equation with discontinuous coefficients, ii) one measurement stationary potential inverse problems for the heat equation with discontinuous coefficients, iii) null controllability for fluid-structure problems in mobile domains and iv) recovering coefficients from locally supported boundary observations for the wave equation. In all the case we explain how to explicitly construct the Carleman weight.
\end{abstract}

Mathematical subject classification: 74G75, 76D05, 93B05.

Key words: Carleman inequalities, exact controllability, inverse problems, Navier-Stokes equations.

\section{Introduction}

Let $\Omega$ be a regular domain in $\mathbb{R}^{n}$ and let us consider a second order adjoint operator of the form $P_{q}^{*} z=f$ evolving in $Q=\Omega \times I$, where $I$ is a time interval. We suppose that $P_{q}$ depends on some stationary parameter $q \in L^{\infty}(\Omega)$. Given

\#696/06. Received: 14/XII/06. Accepted: 14/XII/06.

*This work has been partially supported by grants FONDECYT 1030808, ECOS-CONICYT C04E08 and INRIA-CONICYT. 
some suitable weight function $\Phi$ defined in $Q$, we perform the following change of variables or conjugation:

$$
\begin{gathered}
w=\rho z, \quad \rho=\exp (-s \Phi(x, t)), \quad s>0, \\
P_{q}^{*} z=f \Leftrightarrow \rho P_{q}^{*}\left(\rho^{-1} w\right)=\rho f .
\end{gathered}
$$

For a given parameter $\lambda>0$ and $\alpha$ larger enough, typical weights functions $\Phi$ are of the form:

Heat equation: $P_{q}^{*}=-\delta_{t}-\Delta+q, Q=\Omega \times(0, T)$

$$
\Phi(x, t)=\frac{\exp (\lambda \alpha)-\exp (\lambda \psi(x))}{T-t}
$$

where $\psi(x)$ is some suitable continuous function to be precised later (see for instance Table 1 for some conditions on $\psi$ and Figure 1 for typical shapes of $\psi)$.

Wave equation: $P_{q}^{*}=\delta_{t t}-\Delta+q, Q=\Omega \times(-T, T)$

$$
\Phi(x, t)=-\exp \left(\lambda\left(\psi(x)-\beta t^{2}\right)\right), \psi(x)=\left|x-x_{0}\right|^{2},
$$

where $x_{0}$ is some given point outside $\bar{\Omega}$ and $\beta \in(0,1)$ is suitably chosen.

Schrödinger equation: $P_{q}^{*}=i \partial_{t}+\Delta+q, Q=\Omega \times(-T, T)$

$$
\Phi(x, t)=\frac{\exp (\lambda \alpha)-\exp (\lambda \psi(x))}{(T-t)(T+t)}, \psi(x)=\left|x-x_{0}\right|^{2},
$$

where $x_{0}$ is some given point outside $\bar{\Omega}$.

We also introduce a function $\varphi(x, t)$ such that

$$
\nabla \Phi=-\lambda \nabla \psi \varphi
$$

We consider an internal observational or control region $\omega \subset \subset \Omega$ and a boundary observational or control region $\Gamma_{0} \subset \partial \Omega$. Under some assumptions, we will work with global Carleman inequalities of the form

$$
\begin{gathered}
p_{1}(s, \lambda)\left\|\varphi^{3 / 2} \rho \nabla z\right\|_{L^{2}(Q)}^{2}+p_{0}(s, \lambda)\left\|\varphi^{1 / 2} \rho z\right\|_{L^{2}(Q)}^{2} \\
\leq C\left(\|\rho f\|_{L^{2}(Q)}^{2}+p_{1}(s, \lambda)\left\|\varphi^{3 / 2} \rho \nabla z \cdot n\right\|_{L^{2}\left(\Gamma_{0} \times I\right)}^{2}+p_{0}(s, \lambda)\left\|\varphi^{1 / 2} \rho z\right\|_{L^{2}(\omega \times I)}^{2}\right),
\end{gathered}
$$



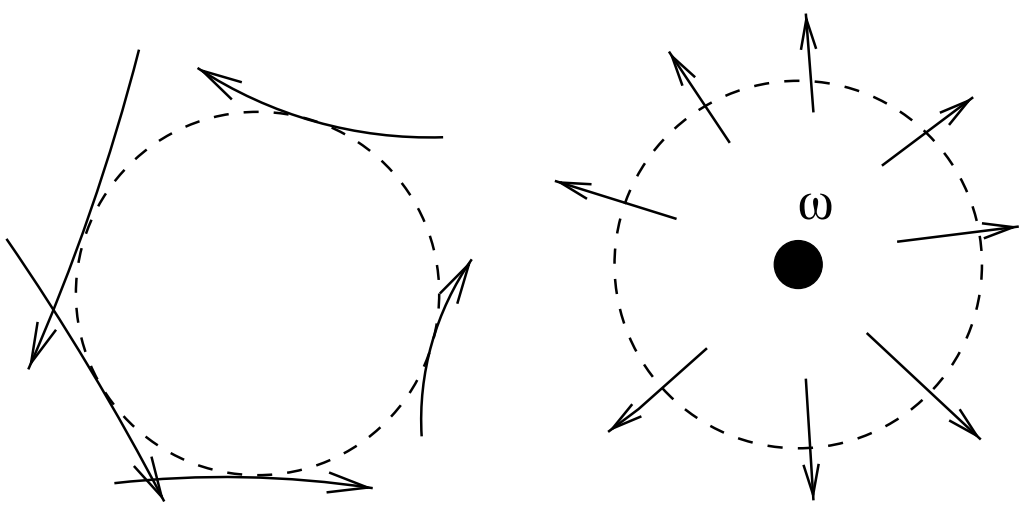

$$
\begin{array}{cc}
p(\cdot, \xi)=\{p, \Phi\}=0, \xi \neq 0 & p(\cdot, \nabla \Phi)=0 \Rightarrow \\
\Rightarrow\{p,\{p, \Phi\}\}>0 & \{\{p, \Phi\}, p(\cdot, \nabla \Phi)\}>0 \\
(\text { cond. } \beta<1) & \text { (cond. }|\nabla \Phi|>0)
\end{array}
$$

Figure 1 - Graphical interpretation of pseudoconvexity for $\Phi=\left|x-x_{0}\right|^{2}-\beta t^{2}$ (waves) with increasing velocity from the outer to the inner levels of $\Phi . p=\xi_{0}^{2}-|\xi|^{2}$ is the principal symbol of $P^{*}$. Left: rays are bicharacteristics, right: arrows are $\nabla \Phi$.

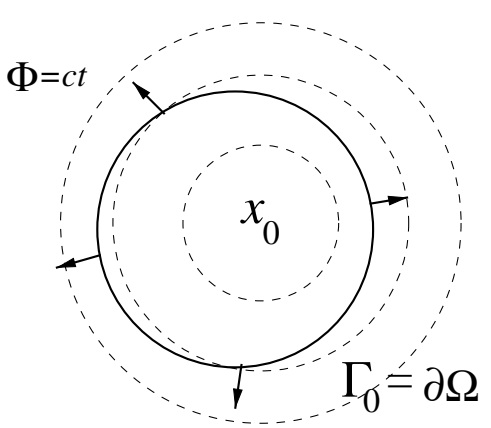

$\nabla \Phi \cdot n<0$ outside $\Gamma_{0}$

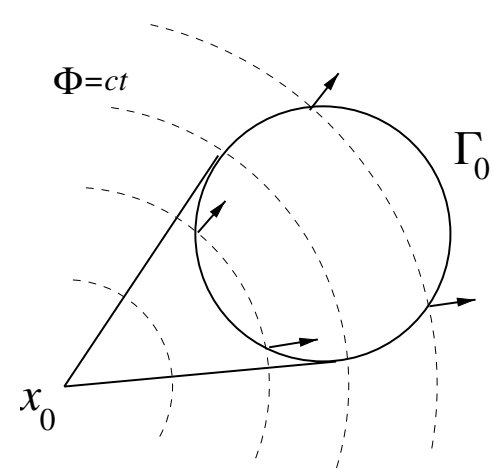

$\nabla \Phi \cdot n<0$ outside $\Gamma_{0}$

$\Gamma_{0}$ is the whole boundary $\Gamma_{0}$ is the exit/lateral part of the boundary

Figure 2 - Graphical interpretation of the strong Lopatinskii condition for $\Phi=\left|x-x_{0}\right|^{2}-\beta t^{2}$ (waves) in the cases that $x_{0}$ is inside and outside of the domain. The level sets of the weight $\Phi$ are represented by the dotted lines.

where $n$ is the unit exterior normal to $\Omega, p_{i}$ are polynomial weights (see Table 2) and $\rho$ is the weight function given by (1). Notice that $\rho \rightarrow 0$ exponentially as $s \Phi \rightarrow+\infty$. 
The internal observational or control region $\omega$ appearing at the right hand side of the global Carleman inequality is such that the pseudoconvexity of $\Phi$ with respect to $P_{q}^{*}$ holds outside $\omega$ (for pseudoconvexity notion see [20], [43]). The boundary observational or control region $\Gamma_{0}$ is such that a strong Lopatinskii condition holds outside $\Gamma_{0}$ (see Table 1 ). If the condition of pseudoconvexity is satisfied in all $\Omega \times I$ then $\omega$ can be empty. Also, if the strong Lopatinskii condition holds on all $\partial \Omega$ then $\Gamma_{0}$ can be empty (see [43] for a much more general statement of global Carleman inequalities in this cases).

\begin{tabular}{c|c|c}
\hline & outside $\omega$ & outside $\Gamma_{0}$ \\
\hline Condition & $|\nabla \psi(x)|>0$ & $\nabla \psi(x) \cdot n<0$ \\
& (necessary to pseudoconvexity) & (strong Lopatinskii) \\
\hline
\end{tabular}

Table 1 - Pseudoconvexity and strong Lopatinskii conditions could not be satisfied in the internal and boundary observational/control regions.

\begin{tabular}{c|cc}
\hline Equation & $p_{1}$ & $p_{0}$ \\
\hline Heat & $s \lambda^{2}$ & $s^{3} \lambda^{4}$ \\
Wave & $s \lambda$ & $s^{3} \lambda^{3}$ \\
Schrödinger & $s \lambda$ & $s^{3} \lambda^{4}$ \\
\hline
\end{tabular}

Table 2 - Polynomial weights in global Carleman inequalities.

Some variants we consider in this review appear when considering operators with discontinuous coefficients in the principal part. In this case, the function $\psi$ has to be well adapted to this new situation and specific global Carleman estimates can be derived. In both cases, some spatial monotonicity of the coefficients is needed. As an application of these inequalities, we study one measurement inverse problems for the heat and wave equations using the general BukhgeimKlibanov approach [11]. The results explained here have been collected from the articles [13], [6], [7] and the preprint [2].

Other interesting variants we consider here arise in the case of mobile domains in fluid-structure problems, when studying the boundary null controllability of an immersed solid into a viscous Navier-Stokes fluid. In this case, the function $\psi$ depends on time, and the global Carleman inequality is much more complicated than (6) because on one hand of incompressibility in Navier-Stokes and on the 
other hand due to the presence of the structure. The results we present in this review were adapted from the articles [9], [10].

The last variant is concerned with one measurement inverse problems from local boundary observations for the wave equation. In this case, the function $\psi$ is modified in order to obtain some strong Lopatinskii condition of the form $\left(x-x_{0}\right) \cdot T n<0$, where $T$ is some linear transformation of the normal field. For further details we refer to the article [14] where the Carleman weights were introduced for two dimensional domains. Here we explain how to deal with the three dimensional case.

Although this is a reduced selection of variants, this collection of Carleman weights and applications illustrate the wealth of the extent of Carleman inequalities when they are applied to the study of some singular inverse and controllability problems.

\section{Inverse source problem for heat transmission problems}

Given $\Omega \subset \mathbb{R}^{n}$ be a bounded and regular subset and let $\Omega_{1}$ be a subdomain such that $\bar{\Omega}_{1} \subset \Omega$ and let us set $\Omega_{0}=\Omega \backslash \bar{\Omega}_{1}$. Let $S$ be the interface between $\Omega_{0}$ and $\Omega_{1}$ with unit normal $n$ exterior to $\Omega_{1}$. Let us denote by $S^{+}$and $S^{-}$the outer and inner sides of the interface $S$ with respect to $n$ and $\Sigma^{+}=S^{+} \times(0, T)$, $\Sigma^{-}=S^{-} \times(0, T)$.

Let us consider the heat transmission problem

$$
\left\{\begin{array}{l}
y_{t}-\operatorname{div}\left(a_{0}(x) \nabla y\right)=f(x) g(x, t) \quad \text { in } \Omega_{0} \times(0, T) \\
y_{t}-\operatorname{div}\left(a_{1}(x) \nabla y\right)=f(x) g(x, t) \quad \text { in } \Omega_{1} \times(0, T) \\
\left.y\right|_{\Sigma^{+}}=\left.y\right|_{\Sigma^{-}},\left.\quad a_{0} \frac{\partial y}{\partial n}\right|_{\Sigma^{+}}=\left.a_{1} \frac{\partial y}{\partial n}\right|_{\Sigma^{-}}, \quad y=0 \text { on } \partial \Omega \times(0, T)(*)
\end{array}\right.
$$

with $a_{i} \geq c_{0}>0$ a.e. in $\Omega$. Let us introduce the space

$$
V=\left\{y \in C^{2}\left(\bar{\Omega}_{i} \times[0, T]\right), i=0,1, y \text { satisfies }(*)\right\} .
$$

The inverse source problem consists in retrieving the source $f(x)$ from the knowledge of $g(x, t)$, the local trace of the solution $y$ in $\omega_{0} \times(0, T)$, where $\bar{\omega}_{0} \subset \Omega_{0}$ and from some time slice $y\left(\cdot, T_{0}\right)$ for some $T_{0} \in(0, T)$, but without any knowledge of the initial condition $y(\cdot, 0)$ of the system. We have to assume also that 
some isotopy type condition is satisfied (see Figure 2 and the details in the article [13]). The inverse stability result is

Theorem 2.1 ([6], [7]). Let $T_{0} \in(0, T)$ and $\omega_{0} \subset \Omega_{0}$ and let us assume that $\Omega_{1}$ and $\Omega_{0}$ satisfy the isotopy type conditions of [13]. Assume that y solution of (7) such that $y, y_{t} \in V$. Assume that $\left.a_{1}\right|_{S^{-}}-\left.a_{0}\right|_{S^{+}} \geq 0$ and that $g \in C^{2}(\bar{\Omega} \times[0, T])$, $\left|g\left(\cdot, T_{0}\right)\right| \geq r_{0}>0$ a.e. in $\Omega$. Then there exists a constant $C=C\left(g, \omega_{0}, T_{0}\right)$ such that for all $f \in L^{2}(\Omega)$

$$
\|f\|_{L^{2}(\Omega)} \leq C\left(\left\|y\left(\cdot, T_{0}\right)\right\|_{H^{2}\left(\Omega_{0}\right)}+\left\|y\left(\cdot, T_{0}\right)\right\|_{L^{2}\left(\Omega_{1}\right)}+\|y\|_{H^{1}\left(0, T ; L^{2}\left(\omega_{0}\right)\right)}\right) .
$$

This result has as main ingredient a global Carleman estimate for the system (7) stated in [13]. This inequality was firstly used in order to prove the exact controllability to trajectories for a semilinear system similar to (7) that is controlled in $\omega_{0} \times(0, T)$. In the general case when $\Omega_{1}$ is not simply connected, and in order to construct the weight functions, an isotopy type condition between $S$ and the boundary of two disjoint open subsets $O_{i}, i=1,2$ of $\Omega_{1}$ is used. Two weights similar to (3) are then constructed of the form

$$
\Phi_{i}(x, t)=\frac{\exp (\lambda \alpha)-\exp \left(\lambda \psi_{i}(x)\right)}{T-t}, i=1,2,
$$

where $\psi_{i} \in V$ and $\nabla \psi=0$ only in $O_{i}$ (see Figure 1 left). Notice that you can also consider the opposite case when $\bar{\Omega}_{0} \subset \Omega$ and $\Omega_{1}=\Omega \backslash \bar{\Omega}_{0}$, and always $\bar{\omega}_{0} \subset \Omega_{0}$. In this case, an isotopy type condition between $\partial \Omega$ and $S$ is a sufficient condition. See Figure 1 right).

\section{Controllability problems in mobile domain for fluid-structure interaction}

Let $\Omega \subset \mathbb{R}^{2}$ be a fixed bounded connected open subset with regular boundary. We denote respectively by $\Omega_{S}(t)$ and $\Omega_{F}(t)=\Omega \backslash \overline{\Omega_{S}(t)}$ the domains occupied by the structure (we consider here only one connected component of solid but the results shown here are still valid for a finite number of solids) and by the fluid respectively. Let $n$ be the unit exterior normal to $\partial \Omega_{S}(t)$. The time evolution for $t \in(0, T)$ of the fluid eulerian velocity $u$ and pressure $p$ is governed by 

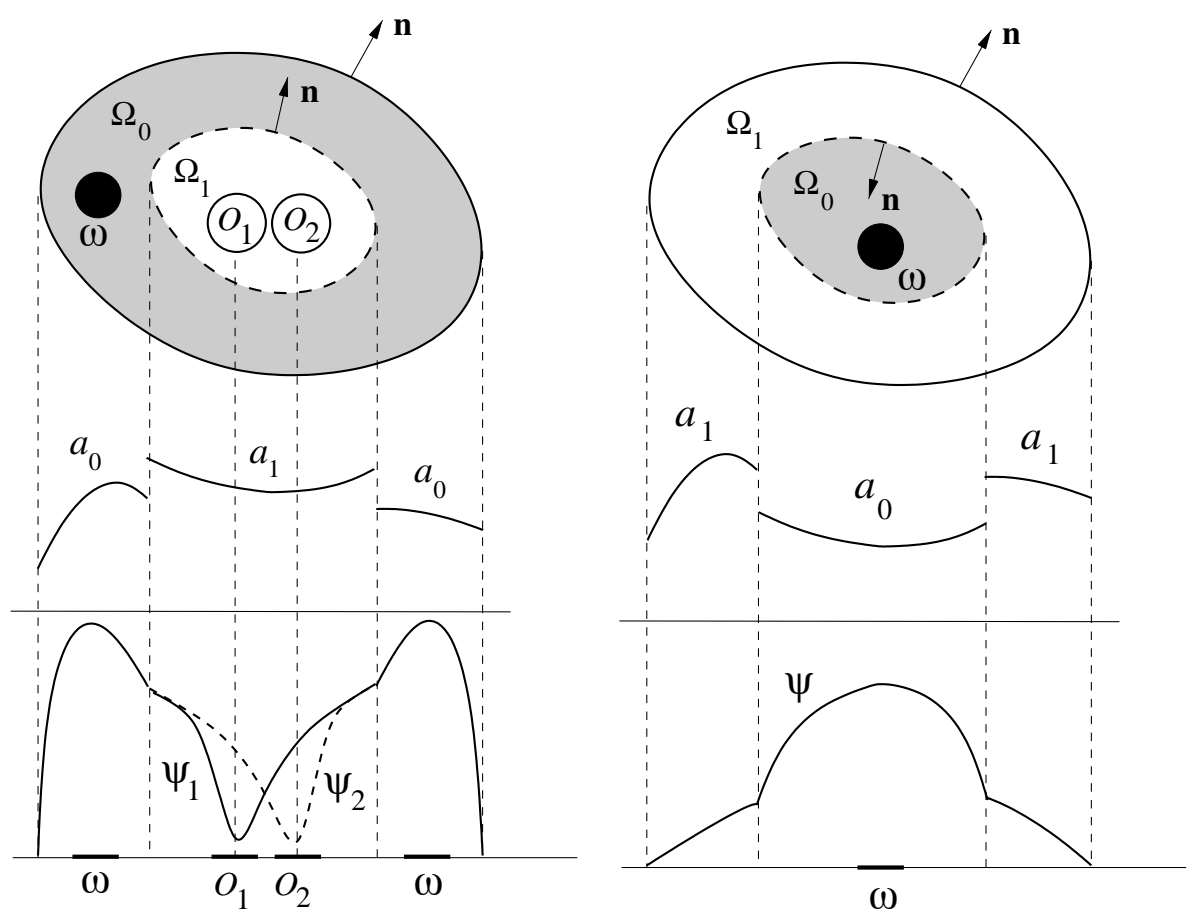

Figure 3 - Construction of the global Carleman weight (bottom curves) for the heat equation with discontinuous coefficients such that $a_{1}\left(S^{-}\right)-a_{0}\left(S^{+}\right)>0$ (middle curves). In the case $\bar{\Omega}_{0} \subset \Omega$ (left) two combined weights are used and in the case $\bar{\Omega}_{1} \subset \Omega$ (right) one weight suffices. In both cases the observation zone $\omega$ is represented by a black dot.

the incompressible Navier-Stokes equations where the Cauchy tensor $\sigma(u, p)=$ $v\left(\nabla u+\nabla u^{t}\right)-p$ Id with viscosity $v>0$ will appear. The movement of the rigid solid with mass $m>0$ and moment of inertia $J>0$ is described by the velocity of its center of mass $a(t) \in \mathbb{R}^{2}$ and by its angular velocity $r(t) \in \mathbb{R}$. The system is

$$
\left\{\begin{array}{l}
\partial_{t} u+(u \cdot \nabla) u-\operatorname{div} \sigma(u, p)=f 1_{\omega}, \operatorname{div} u=0 \text { in } \Omega_{F}(t) \\
m \ddot{a}=\int_{\partial \Omega_{S}(t)} \sigma(u, p) n d \sigma, J \dot{r}=\int_{\partial \Omega_{S}(t)}(\sigma(u, p) n) \cdot(x-a)^{\perp} d \sigma, \\
u=\dot{a}+r(x-a)^{\perp} \text { on } \partial \Omega_{S}(t), u=0 \text { on } \partial \Omega, \\
u(0, \cdot)=u_{0} \text { in } \Omega_{F}(0), a(0)=a_{0}, \dot{a}(0)=a_{1}, r(0)=r_{0},
\end{array}\right.
$$




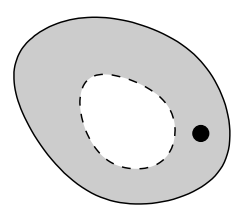

(a)

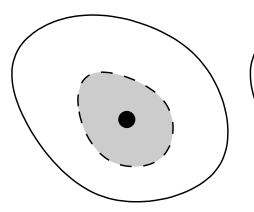

(a)

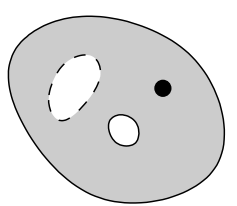

(b)

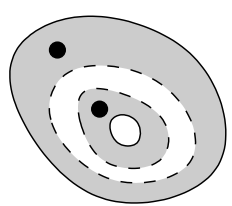

(c)

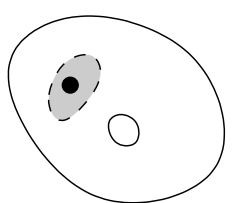

(b)

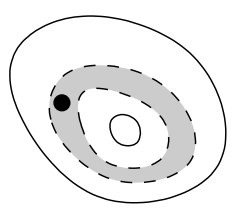

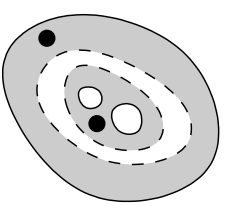

(d)

(c)

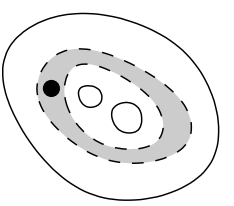

(d)

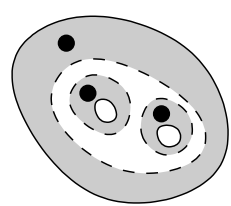

(e)

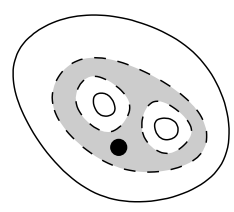

(e)

Figure $4-$ In all the figures $\Omega_{0}$ is filled in gray and the observation region $\omega$ is represented by a black dot. The interface $S$ between $\Omega_{0}$ and $\Omega_{1}$ is represented by a dashed line and the boundary $\partial \Omega$ by a solid line. In the lower line, all the examples except for (b) and (d) satisfy the isotopy type condition of [13] between $S$ and $\partial \Omega$. In the upper line, all the examples except for (e) satisfy the isotopy type condition between $S$ and the boundaries of two disjoints subsets $O_{1}$ and $O_{2}$ of $\Omega_{1}$. In all the exceptions, the Carleman weight can not be constructed with the method of [13].

Here the function $f$ is the control function which acts over a fixed small nonempty open subset $\omega$ (with characteristic function $1_{\omega}$ ). See Figure 5 .

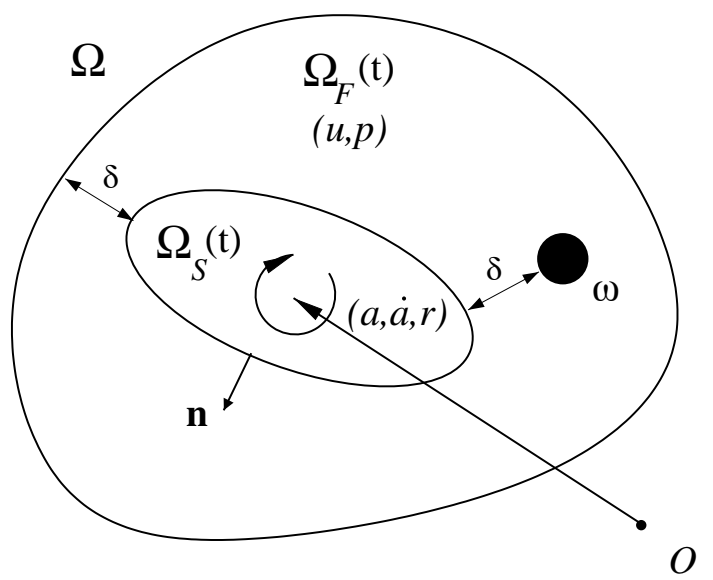

Figure 5 - Notations of Section 3. 
We have used the notation $x^{\perp}=\left(x_{1}, x_{2}\right)^{\perp}=\left(-x_{2}, x_{1}\right)$. The total angle $\theta$ associated to the angular velocity $r$ is defined by $\theta(t)=\theta_{0}+\int_{0}^{t} r(s) d s$, where $\theta_{0} \in \mathbb{R}$ complements the initial data. The existence of solutions and regularity for this system has been recently studied in several papers (see [39], [41] and the references therein).

The controllability result is the following, saying that it is possible to drive the structure and the fluid at rest and the immersed solid up to its reference position in arbitrarily small time with a localized control $f$, provided the initial conditions are sufficiently small.

Theorem 3.1 ([9]). Suppose that: $i$ ) the initial body solid shape satisfies

$\Omega_{S}(0) \subset \Omega \backslash \omega, d\left(\Omega_{S}(0), \partial(\Omega \backslash \omega)\right)>0, \int_{\partial \Omega_{S}(0)}\left(y-a_{0}\right) d \sigma=0 ;$

ii) the initial conditions $u_{0} \in H^{3}\left(\Omega_{F}(0)\right)^{2}, a_{0} \in \mathbb{R}^{2}, a_{1} \in \mathbb{R}^{2}, \theta_{0} \in \mathbb{R}$ and $r_{0} \in \mathbb{R}$ satisfy the compatibility conditions

$$
\begin{aligned}
& \operatorname{div} u_{0}=0 \text { in } \Omega_{F}(0), \\
& u_{0}=a_{1}+r_{0}\left(x-a_{0}\right)^{\perp} \text { on } \partial \Omega_{S}(0) \text { and } \\
& u_{0}=0 \text { on } \partial \Omega
\end{aligned}
$$

iii) the acceleration $u_{1}$ of the fluid and the accelerations $a_{2}$ and $r_{1}$ of the structure at initial time (determined by the equations of the motion (11) and by the boundary conditions taken at initial time, well defined thanks to Helmholtz decomposition) satisfy

$$
\begin{aligned}
u_{1}= & 0 \text { on } \partial \Omega, \\
u_{1}= & a_{2}+r_{1}\left(x-a_{0}\right)^{\perp}-r_{0}^{2}\left(x-a_{0}\right) \\
& -\nabla u_{0}\left(a_{1}+r_{0}\left(x-a_{0}\right)^{\perp}\right) \text { on } \partial \Omega_{S}(0) .
\end{aligned}
$$

Under the above assumptions, for all $T>0$ there exists $\varepsilon>0$ and $f \in$ $L^{2}((0, T) \times \omega)^{2}$ such that if

$$
\left\|u_{0}\right\|_{H^{3}\left(\Omega_{F}(0)\right)^{2}}+\left|a_{0}\right|+\left|a_{1}\right|+\left|\theta_{0}\right|+\left|r_{0}\right| \leq \varepsilon
$$

then

$$
u(T, \cdot)=0 \text { in } \Omega_{F}(T), a(T)=0, \dot{a}(T)=0, \theta(T)=0, r(T)=0 .
$$


The last condition in (12) is a symmetry restriction over the shape of the solid needed in the proof of the Carleman inequality satisfied by the adjoint problem of the linearized system to get estimates on the structure motion from estimates on the fluid velocity on the interface.

The result only holds for small initial data because we want to keep the noncollision condition on the whole interval $(0, T)$

$$
\inf _{t \in(0, T)} \mathrm{d}\left(\Omega_{S}(t), \partial(\Omega \backslash \omega)\right)>0 .
$$

The first result of this kind using global Carleman estimates was obtained in [12] for a one-dimensional Burgers-particle system studied in [45]. Also, similar results to the one presented here has been simultaneously and independently obtained in the preprint [12] (see the preprint version of [10]).

The idea of the proof is the following and follows ideas for the controllability of the Navier-Stokes equations recently used in [15], [21] and the ideas of [12]. We first consider a linearized problem. Let $(\tilde{a}, \tilde{r})$ be given in $H^{2}(0, T)^{2} \times H^{1}(0, T)$. We define $\tilde{\theta}$ the angle associated to the rotation velocity $\tilde{r}$ defined up to a constant. For any $t \in(0, T)$, we define the structure domain

$$
\widetilde{\Omega}_{S}(t)=\left\{\tilde{a}(t)+R_{\tilde{\theta}(t)-\theta_{0}}\left(y-a_{0}\right), y \in \Omega_{S}(0)\right\} .
$$

We suppose that $\tilde{a}(0)=a_{0}, \tilde{\theta}(0)=\theta_{0}$ and $\inf _{t \in(0, T)} \mathrm{d}\left(\widetilde{\Omega}_{S}(t), \partial(\Omega \backslash \omega)\right)>0$. Thus, we can define the fluid domain $\widetilde{\Omega}_{F}(t)=\Omega \backslash \widetilde{\Omega}_{S}(t)$. We also consider a given velocity $\tilde{u}$ satisfying regularity properties and compatibility conditions with $(\tilde{a}, \tilde{r})$. The linearized problem associated to (11) is the one where we have replaced in $(11) \Omega_{S}(t)$ by $\widetilde{\Omega}_{S}(t), \Omega_{F}(t)$ by $\widetilde{\Omega}_{F}(t)$ and the nonlinear term $(u \cdot \nabla) u$ in the first equation by $(\tilde{u} \cdot \nabla) u$. We prove a null controllability result for the linearized problem with the help of a Carleman inequality shown on the adjoint system associated to a linearized system. Finally, Theorem 3.1 is proved by applying Kakutani's fixed point theorem.

We will give the Carleman inequality here for two reasons. First, once NavierStokes equations are involved, the corresponding global Carleman inequality is different from (6) because of the pressure (or because of incompressibility). Indeed, the exponential weights appearing at the left and right hand sides of the inequality are no more the same. Secondly, since we are working with variable domains, this is in fact a Carleman inequality in moving domains. 
Let us first introduce the corresponding adjoint operator $P_{q}^{*}$ on this case given by the solution $(v, q, b, \gamma)$ of the linear system

$$
\left\{\begin{array}{l}
-\partial_{t} v-(\tilde{u} \cdot \nabla) v-\operatorname{div} \sigma(v, q)=0, \operatorname{div} v=0 \text { in } \widetilde{\Omega}_{F}(t), \\
m \ddot{b}(t)=-\int_{\partial \widetilde{\Omega}_{S}(t)} \sigma(v, q) n d \sigma, \\
J \dot{\gamma}(t)=-\int_{\partial \widetilde{\Omega}_{S}(t)}(\sigma(v, q) n) \cdot(x-\tilde{a}(t))^{\perp} d \sigma, \\
v=\dot{b}+\gamma(x-\tilde{a})^{\perp} \text { on } \partial \widetilde{\Omega}_{S}(t), v=0 \text { on } \partial \Omega, \\
v(T, \cdot)=v_{0}^{T} \text { in } \widetilde{\Omega}_{F}(T), b(T)=0, \dot{b}(T)=b_{1}^{T}, \gamma(T)=\gamma_{0}^{T},
\end{array}\right.
$$

with

$$
\begin{aligned}
& \operatorname{div} v_{0}^{T}=0 \text { in } \widetilde{\Omega}_{F}(T), \\
& v_{0}^{T}=b_{1}^{T}+\gamma_{0}^{T}(x-\tilde{a}(T))^{\perp} \text { on } \partial \widetilde{\Omega}_{S}(T) \quad \text { and } \\
& v_{0}^{T}=0 \text { on } \partial \Omega
\end{aligned}
$$

and $\tilde{u}$ regular enough and conveniently chosen.

The following extremal weights appear when eliminating the explicit dependence on the pressure $q$ of the Carleman inequality. For a given field $v(x, t)$ we take the notation:

$$
\bar{v}(t)=\sup _{x \in \widetilde{\Omega}_{F}(t)} v(x, t), \quad \underline{v}(t)=\inf _{x \in \widetilde{\Omega}_{F}(t)} v(x, t)
$$

Theorem 3.2 ([9]). There exists $\hat{s}, \hat{\lambda}$ and $C$ depending on $\Omega, \omega$ and $T$ such that, for every regular solution $(v, b, \gamma)$ of $(15)$, for all $\lambda>\hat{\lambda}$ and $s>\hat{s}$ we have:

$$
\begin{aligned}
& \int_{0}^{T} \int_{\widetilde{\Omega}_{F}(t)} \rho^{2}\left(\frac{1}{s \varphi}\left(|\Delta v|^{2}+\left|\partial_{t} v\right|^{2}\right)+s \lambda^{2} \varphi|\nabla v|^{2}+s^{3} \lambda^{4} \varphi^{3}|v|^{2}\right) d x d t \\
& \quad+s \lambda \int_{0}^{T} \underline{\rho}^{2} \underline{\varphi}\left(|\ddot{b}|^{2}+|\dot{\gamma}|^{2}\right) d t \\
& \int_{0}^{T} \int_{\partial \widetilde{\Omega}_{S}(t)} \underline{\rho}^{2}\left(s^{3} \lambda^{3} \underline{\varphi}^{3}|v|^{2} \nabla \psi \cdot n+2 s \underline{\varphi} \underline{\varphi}|\nabla v n|^{2}\right) d \sigma d t \\
& \quad \leq C s^{19 / 2} \lambda^{13} \int_{0}^{T} \int_{\omega}(\bar{\rho} / \underline{\rho})^{2} \bar{\rho}^{2} \bar{\varphi}^{10}|v|^{2} d x d t .
\end{aligned}
$$

The Carleman inequality is expressed on the moving domains $\widetilde{\Omega}_{S}(t)$ and $\widetilde{\Omega}_{F}(t)$ and the transport theorem is used on its deduction. More precisely, the weight 
$\Phi$ used here is of the form (compare with (3))

$$
\Phi(x)=\frac{\exp \left(\lambda \alpha_{0}\right)-\exp \left(\alpha_{1} \lambda \psi(x, t)\right)}{(T-t)^{4}}
$$

where $\alpha_{0}, \alpha_{1}$ are suitable constants. The time dependent weight function $\psi(x, t)$ is chosen as the standard weight for the heat equation but it follows the shape of $\widetilde{\Omega}_{S}(t)$. More precisely, $\psi(x, t)$ is a regular function such that $\frac{\partial \psi}{\partial n}<0$ on $\Sigma$, $|\nabla \psi|>0$ outside $\omega$ and it satisfies the time dependent conditions $\frac{\partial \psi}{\partial n}>0$ on $\partial \widetilde{\Omega}_{S}(t), \psi$ constant on $\partial \widetilde{\Omega}_{S}(t)$. Notice that an interesting property is that the spatial gradient of $\psi$ is related to $1 / \delta(t)$, where $\delta(t)>0$ is the distance between $\widetilde{\Omega}_{S}(t)$ and $\partial(\Omega \backslash \omega)$. This could be useful when doing explicit calculations of constants in the Carleman inequality as the collision parameter $\delta(t) \rightarrow 0^{+}$as $t \rightarrow t_{0}^{-}$for some collision time $t_{0}>0$.

\section{Inverse problem in wave equation with partial boundary data}

The main idea is to modify the weight function $\Phi$ given in (4) in such a way that its gradient $\nabla \Phi$ a rotation of the original field $\left(x-x_{0}\right)$ with a radially dependent magnitude. This concept come up from multipliers technique and controllability [19], [34].

Let $\Omega$ be a domain in $\mathbb{R}^{n}, n=2,3$. In order to solve the Dirichlet to Neumann one measurement inverse problem, it suffices to measure on a rotated exit part of the boundary $\Gamma_{r}$. If $n=2$, this region depends on a point $x_{0} \in \mathbb{R}^{n}$ and on a rotation $T_{\theta}$ in an angle $\theta \in(-\pi / 2, \pi / 2)$. If $n=3$, it depends also on a unit direction $\alpha \in \mathbb{R}^{3}$ and the rotation $T_{\theta}$ is considered on the orthogonal plane to $\alpha$ denoted here by $\alpha^{\perp}$. We will use the notation $v^{\perp}=v-(v \cdot \alpha) \alpha$ for the projection of the field $v$ on $\alpha^{\perp}$. More precisely

$$
\begin{aligned}
& (n=2) \Gamma\left(x_{0}, \theta\right)=\left\{x \in \partial \Omega \mid\left(x-x_{0}\right) \cdot T_{\theta} n>0\right\} \\
& (n=3) \Gamma\left(x_{0}, \alpha, \theta\right)=\left\{x \in \partial \Omega \mid\left(x-x_{0}\right) \cdot\left(\cos \theta\left(n-n^{\perp}\right)+T_{\theta} n^{\perp}>0\right\}\right.
\end{aligned}
$$

We can always reduce the two-dimensional case to the bi-dimensional one by considering $\mathbb{R}^{2}$ immersed in $\mathbb{R}^{3}$ with $\alpha=(0,0,1)$, so in the following we will only refer to the three-dimensional case. Notice that, as $\theta$ approaches $\pi / 2$, the rotated exit part tends to be the union of the boundary points $x$ where the 
dot product between the vector $x-x_{0}$ and the projection $n^{\perp}$ rotated in $\pi / 2$ is positive (see Figure 6). There are of course an infinite number of intermediate cases depending on the localization of $x_{0}$, the direction of $\alpha$ and the angle $\theta$. The case $\theta=0$ corresponds to the standard exit condition previously used for the same inverse problem [24]. Remark also that the rotated exit condition is a particular case of the geometrical optics BLR condition [1], [33].

The main stability result is the following (we present here the case $x_{0} \notin \bar{\Omega}$, the other case in which two arbitrarily near interior points are used can be found in [14]).

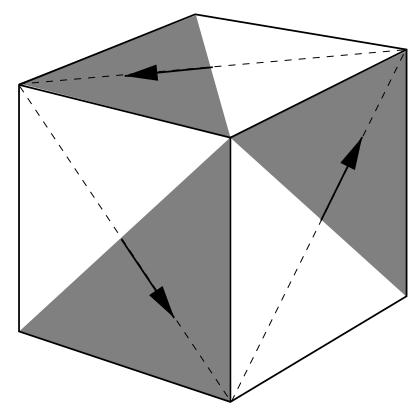

Figure 6 - Rotated exit boundary region in a cube $(-a, a)^{3}$ with respect to the origin $x_{0}=0$ and a diagonal axis $\alpha$ parallel to $(1,1,1)$ with orthogonal plane $\alpha^{\perp}$ (dotted lines). The exit region (shaded) are the boundary points whose position vectors have a positive dot product with the $\pi / 2$ rotation of the projection of the normal $n^{\perp}$ on $\alpha^{\perp}$ (arrows). By taking a suitable time-dependent Neumann measure on a little bit more than this region, the inverse problem of recovering a time-independent potential in the wave equation is locally stable (not really in a cube but in a regularized $C^{2}$ cube).

Theorem 4.1 ([14]). Let $P_{q}^{*}=\partial_{t t}-\Delta+q$ and let $u(q)$ and $u(\bar{q})$ be the respective solutions of $P_{q}^{*} u=0$ with Dirichlet boundary conditions associated to $q, \bar{q} \in L^{\infty}(\Omega)$ and with Neumann measurements $\xi$ and $\bar{\xi}$ on $\Gamma_{r} \times(0, T)$ respectively. There exists a time $\bar{T}>0$ such that if $T>\bar{T}$, if $u(\bar{q}) \in H^{1}\left(0, T ; L^{\infty}(\Omega)\right)$ and if $|u(0)| \geq \alpha_{0}>0$ a.e. in $\Omega$, then there exists a positive constant $C_{M}$ depending on $M=\|q\|_{L^{\infty}(\Omega)}$ such that

$$
\|\bar{q}-q\|_{L^{2}(\Omega)} \leq C_{M}\|\bar{\xi}-\xi\|_{H^{1}\left(0, T ; L^{2}\left(\Gamma_{r}\right)\right)} \quad \forall q \text { with }\|q\|_{L^{\infty}(\Omega)} \leq M .
$$


If $\theta \rightarrow \pm \frac{\pi}{2}$ then $\bar{T} \rightarrow+\infty$ and it behaves asymptotically as

$$
\bar{T} \approx \frac{R_{0}}{\sqrt{\beta}} \exp \left(\frac{2}{\cos \theta} \theta_{0}\right)
$$

where $R_{0}=\sup _{x \in \Omega}\left|x-x_{0}\right|$ and $\theta_{0}=\sup _{x \in \Omega} \arg \left(x-x_{0}\right)^{\perp}-\inf _{x \in \Omega} \arg \left(x-x_{0}\right)^{\perp}$, i.e., the angle of view of $\Omega$ with respect to the axis of direction $\alpha$ passing by $x_{0}$.

The proof of this Theorem is based on a global Carleman estimate using the variant of the Carleman weight for the wave equation (compare with (4))

$$
\Phi(x, t)=-\lambda \exp \left(\cos \theta\left|x-x_{0}\right|^{2} \exp \left(2 \tan \theta \arg \left(x-x_{0}\right)^{\perp}\right)-\beta t^{2}\right)
$$

for some suitable constant $\beta \in(0,1)$. This weight was constructed in order to make appear in the gradient $\nabla \Phi$ the matrix

$$
\left(\begin{array}{ccc}
\cos \theta & 0 & 0 \\
0 & \cos \theta & \sin \theta \\
0 & -\sin \theta & \cos \theta
\end{array}\right)
$$

written in a basis attached to $\alpha, \alpha^{\perp}$. The product of this matrix and a vector field $v$ is exactly $\cos \theta\left(v-v^{\perp}\right)+T_{\theta} v^{\perp}$, expression which appears in the definition (21) of $\Gamma_{r}$.

The main steps in the deduction of such inequality are taken from [35, 23] following a well known technique due to Bukhgeim and Klibanov [11], [29]. Roughly speaking, the technique in this case consists in reducing the problem to a source inverse problem for the perturbed equation around $u(\bar{q})$ and then take its time derivative in order to obtain a quasi-observability inequality after use of the Carleman inequality. Quasi, since the initial condition is bounded not only by the observation but also by the unknown source term, but a sufficiently large time and the properties of the Carleman weight allow to get rid of this source term.

There are also geometrical exit type conditions for the analogous inverse problem in the case of the Scrhödinger equation (see [3]). The present method should also work in this case because the spatial part used in Carleman weights for wave and Schödinger equations are the same (compare (4) with (5)). Nevertheless, in the case of the Scrhödinger operator, it is strongly possible that the geometrical 
optic condition are not necessary to solve the one measurement inverse problem (see [27]). Other completely different problem is the case when you have Dirichlet to Newmann map measurements. In this case, a suitable arbitrarily small boundary of measurements is enough to solve the inverse problem both for Schödinger and wave equations (see [28]). But recently, it has been shown that you can solve the one measurement problem for the wave equation with an arbitrarily boundary measurement region (in the case of Neumann boundary conditions and Dirichlet measurements), but the corresponding inequality analogous to (22) is logarithmic [5].

\section{Inverse coefficient problem for wave transmission problems}

Notice that recently, Global Carleman estimates and application to one measurement inverse problems for the wave equation were obtained in the case of variable but still regular coefficients [4], [26]. The inverse problem of retrieving coefficients from a wave equation with discontinuous coefficients from boundary measurements arise naturally in geophysics and more precisely, in seismic prospection of earth inner layers [44].

Let $\Omega$ and $\Omega_{1} \subset \Omega$ be two open subsets of $\mathbb{R}^{2}$ with smooth boundaries $\Gamma$ and $\Gamma_{1}$ respectively and let $\Omega_{2}=\Omega \backslash \bar{\Omega}_{1}$. To fix ideas we assume that $\Omega_{1}$ is simply connected. We set:

$$
\bar{a}(x)= \begin{cases}a_{1} & x \in \Omega_{1} \\ a_{2} & x \in \Omega_{2}\end{cases}
$$

with $a_{j}>0$ for $j=1,2$, for each $q \in L^{\infty}(\Omega)$, we consider $u(q)$ as the solution of the following wave transmission equation

$$
\left\{\begin{array}{l}
u_{t t}-\operatorname{div}(\bar{a}(x) \nabla u)+q(x) u=0 \text { in } Q=\Omega \times(0, T) \\
u=g \text { on } \Sigma=\Gamma \times(0, T) \\
u(0)=u_{0} \text { in } \Omega \\
u_{t}(0)=u_{1} \text { in } \Omega .
\end{array}\right.
$$

The following inverse stability result holds (see the preprint [2]):

Theorem 5.1 ([2]). Assume $\Omega_{1}$ is strictly convex and $a_{1}>a_{2}>0$. Let $\mathcal{U}$ be a bounded subset of $L^{\infty}(\Omega), \bar{q} \in L^{\infty}(\Omega)$ and $r>0$. If $\left|u_{0}(x)\right| \geq r>0$ a. e. in $\Omega$ 
and $u(\bar{q}) \in H^{1}\left(0, T ; L^{\infty}(\Omega)\right)$, then there exists $C=C\left(\Omega, T,\|q\|_{L^{\infty}(\Omega)}, \mathcal{U}\right)>$ 0 such that:

$$
\|\bar{q}-q\|_{L^{2}(\Omega)} \leq C\left\|\frac{\partial u(\bar{q})}{\partial n}-\frac{\partial u(q)}{\partial n}\right\|_{H^{1}\left(0, T ; L^{2}(\Gamma)\right)}
$$

for all $u_{0} \in H_{0}^{1}(\Omega)$ and $q \in \mathcal{U}$.

This Theorem is proved by combining the Carleman inequality for the wave equation with discontinuous coefficients proved in [2] and the method of Bukhgeim-Klibanov explained in section 4. To this end, system (27) is viewed as two wave equations with constant coefficients coupled with transmission conditions (see [31]). Then, a global Carleman inequality is found out for this transmission problem by working with variants of Carleman weights of the form (compare with (4))

$$
\begin{gathered}
\Phi(x, t)=-\exp (\lambda \varphi(x)), \\
\varphi(x)=\left\{\begin{array}{l}
\eta(x) \frac{a_{2}}{r(x)^{2}}\left|x-x_{0}\right|^{2}-\beta t^{2}+M_{1} \quad \text { in } \Omega_{1} \times(-T, T) \\
\frac{a_{1}}{r(x)^{2}}\left|x-x_{0}\right|^{2}-\beta t^{2}+M_{2} \quad \text { in } \Omega_{2} \times(-T, T)
\end{array}\right.
\end{gathered}
$$

where $M_{1}$ and $M_{2}$ are constants such that $M_{1}-M_{2}=a_{1}-a_{2}, r(x)=\left|x_{0}-y(x)\right|$, $y(x)=\Gamma_{1} \cap\left[x_{0}, x\right]$ and $\eta$ is some cut-off function with support in $\Omega_{1}$ centered at $x_{0}$. We also combine the Carleman inequalities obtained from two different interior points as we did in section 2, see also Figure 1, left. The convexity hypothesis on $\Omega_{1}$ comes from the fact that the positiveness of the Hessian of the weight $\Phi$ is related with the curvature of $\Gamma_{1}$ with respect to $x_{0}$.

There are a lot of important works concerning this inverse problem in the case that a wide class of measurements are available. In these cases, microlocal analysis has been used and it gives positive answer to the problem of retrieving coefficients and discontinuity interfaces without restrictive hypothesis of convexity of the interfaces or monotonicity of the speed of waves. These kinds of results are fundamental for seismic prospection. For an overview on this subject see [44] and the references therein, in particular related to thie study are the works of [8], [17], [37], [38], [40]. 


\section{REFERENCES}

[1] C. Bardos, G. Lebeau and J. Rauch, Sharp sufficient conditions for the observation, control and stabilization of waves from the boundary. SIAM J. Contr. Optim., 30 (1992), 1024-1465.

[2] L. Baudouin, A. Mercado and A. Osses, Global Carleman estimates in a transmission problem for the wave equation. Application to a one-measurement inverse problem, Inverse Problems, 23 (2007), 1-22.

[3] L. Bauduoin and J.-P. Puel, Uniqueness and stability in an inverse problem for the Schödinger equation. Inverse Problems, 18(6) (2002), 1537-1554.

[4] M. Bellassoued, Uniqueness and stability in determining the speed of propagation of secondorder hyperbolic equation with variable coefficients. Appl. Anal., 83(10) (2004), 983-1014.

[5] M. Bellassoued and M. Yamamoto, Logarithmic stability in determination of a coefficient in an acoustic equation by arbitrary boundary observation. J. Math. Pures Appl., 85 (2006), 193-224.

[6] M. Bellassoued and M. Yamamoto, Inverse source problem for a transmission problem for a parabolic equation. J. Inv. Ill-Posed Problems, 14(1) (2006), 47-56.

[7] A. Benabdallah, P. Gaitan and J. Le Rousseau, Stability of discontinuous diffusion coefficients and initial conditions in an inverse problem for the heat equation. Preprint LATP - Laboratoire d'Analyse, Topologie, Probabilités, CNRS: UMR 6632, Université Aix-Marseille I, France, May 2006.

[8] G. Beylkin, The inversion problem and applications of the generalized Radon transform. Comm. Pure Appl. Math., 37(5) (1984), 579-599.

[9] M. Boulakia and A. Osses, Two-dimensional local null controllability of a rigid structure in a Navier-Stokes fluid. C.R. Math. Acad. Sci. Paris., Ser. I, 343(2) (2006) 105-109.

[10] M. Boulakia and A. Osses, Two-dimensional local null controllability of a rigid structure in a Navier-Stokes fluid. Preprint 139, U. de Versailles Saint-Quentin, France, October 2005. To appear in ESAIM-COCV.

[11] A. L. Bukhgeim and M. V. Klibanov, Global uniqueness of a class of inverse problems. Dokl. Akad. Nauk SSSR, 260(2) (1981), 269-272, English translation: Soviet Math. Dokl, 24(2) (1982), 244-247.

[12] A. Doubova and E. Fernandez-Cara, Local and global controllability results for simplified one-dimensional models of fluid-solid interaction, to appear in Math. Models Methods Appl. Sci.

[13] A. Doubova, A. Osses and J.-P. Puel, Exact controllability to trajectories for semilinear heat equations with discontinuous diffusion coefficients. A tribute to J. L. Lions. ESAIM Control Optim. Calc. Var., 8 (2002), 621-661.

[14] A. Doubova and A. Osses, Rotated weights in global Carleman estimates applied to an inverse problem for the wave equation. Inverse Problems, 22 (2006), 265-296. 
[15] E. Fernandez-Cara, S. Guerrero, O. Yu. Imanuvilov and J.-P. Puel, Local exact controllability of the Navier-Stokes system. J. Math. Pures Appl., 83(12) (2004), 1501-1542.

[16] A. Fursikov and O. Yu. Imanuvilov, Controllability of Evolution Equations. Lecture Notes, 34, Seoul National University, Korea, 1996.

[17] S. Hansen, Solution of a hyperbolic inverse problem by linearization. Comm. Partial Differential Equations, 16(2-3) (1991), 291-309.

[18] A. Haraux, Séries lacunaires et contrôle semi-interne des vibrations d'une plaque rectangulaire. J. Math. pures et appl., 68(4) (1989), 457-465.

[19] L. Ho, Observabilité frontière de l'équation des ondes. (Boundary observability of the wave equation). C.R. Acad. Sci., Paris, Ser. I, 302 (1986), 443-446.

[20] L. Hörmander, The analysis of linear partial differential operators II. Springer-Verlag, Berlin, 1983.

[21] O. Yu. Imanuvilov, Remarks on exact controllability for the Navier-Stokes equations. ESAIM Control Optim. Calc. Var., 6 (2001), 39-72.

[22] O. Yu. Imanuvilov and J.-P. Puel, Global Carleman estimates for weak solutions of elliptic nonhomogeneous Dirichlet problems. Internat. Math. Res. Notices, 16 (2003), 883-913.

[23] O. Yu. Imanuvilov, On Carleman estimates for hyperbolic equations. Asymptot. Anal., 32(3-4) (2002), 185-220.

[24] O. Yu. Imanuvilov and M. Yamamoto, Global uniqueness and stability in determining coefficients of wave equations. Commun. Partial Diff. Eqns, 26(7-8) (2001), 1409-1425.

[25] O. Yu. Imanuvilov and M. Yamamoto, Global lipschitz stability in an inverse hyperbolic problem by interior observations. Inverse Problems, 17(4) (2001), 717-728.

[26] O. Yu. Imanuvilov and M. Yamamoto. Determination of a coefficient in an acoustic equation with a single measurement. Inverse Problems, 19(1) (2003), 157-171.

[27] S. Jaffard, Contrôle interne exact des vibrations d'une plaque rectangulaire, Portugal. Math., 47 (1990), 423-429.

[28] C. Kenig, J. Sjöstrand and G. Uhlmann, The Calderón problem with partial data, to appear.

[29] M. V. Klibanov and J. Malinsky, Newton-Kantorovich method for three-dimensional potential inverse scattering problem and stability of the hyperbolic Cauchy problem with timedependent data. Inverse Problems, 7 (1991), 577-595.

[30] V. Komornik, Exact controllability and stabilization. The multiplier method, Research in Applied Mathematics 36, Paris, Masson, 1994.

[31] J.-L. Lions, Contrôlabilité exacte, perturbation et stabilisation de Systèmes Distribués, 1, Masson, Paris, 1988.

[32] J.-L. Lions and E. Magenes, Non-homogeneous Boundary Value Problems and applications, Springer-Verlag, Berlin, 1972.

Comp. Appl. Math., Vol. 25, N. 2-3, 2006 
[33] L. Miller, Escape function conditions for the observation, control, and stabilization of the wave equation. SIAM J. Control Optim., 41(5) (2003), 1554-1566.

[34] A. Osses, A rotated multiplier applied to the controllability of waves, elasticity, and tangential Stokes control. SIAM J. Control and Optim., 40(3) (2001), 777-800.

[35] J.-P. Puel, Application of Carleman Inequalities to Controllability and Inverse problems, Textos de Metodos Matematicos de l'Instituto de Matematica de l'UFRJ, to appear.

[36] J.-P. Puel and M. Yamamoto, Generic well posedness in a multidimensional hyperbolic inverse problem, J. of Inverse and ill-posed problems, 1 (1997), 53-83.

[37] Rakesh, A linearised inverse problem for the wave equation. Comm. Partial Differential Equations, 13(5) (1988), 573-601.

[38] Rakesh, An inverse impedance transmission problem for the wave equation. Comm. Partial Differential Equations 18(3-4) (1993), 583-600.

[39] J. San Martín, V. Starovoitov and M. Tucsnak, Global weak solutions for the two dimensional motion of several rigid bodies in an incompressible viscous fluid. Arch. Rational Mech. Anal., 161(2) (2002), 113-147.

[40] W.W. Symes, A differential semblance algorithm for the inverse problem of reflection seismology. Comput. Math. Appl., 22(4-5) (1991), 147-178.

[41] T. Takahashi, Analysis of strong solutions for the equations modeling the motion of a rigidfluid system in a bounded domain. Adv. Differential Equations, 8(12) (2003), 1499-1532.

[42] T. Takahashi and O. Yu Imanuvilov, Exact controllability of a fluid-rigid body system, Preprint IECN, U. Nancy, France, november 2005.

[43] D. Tataru, Carleman estimates and unique continuation for solutions to boundary value problems. J. Math. Pures Appl., (9) 75(4) (1996), 367-408.

[44] M. de Hoop, Microlocal analysis of seismic in inverse scattering. Inside out: in inverse problems and applications, 219-296. Math. Sci. Res. Inst. Publ., 47 (2003), Cambridge Univ. Press, Cambridge.

[45] J.L. Vázquez and E. Zuazua, Lack of collision in a simplified 1-dimensional model for fluid-solid interaction, M3AS, 16(5) (2006), 637-678.

[46] M. Yamamoto, Uniqueness and stability in multidimensional hyperbolic inverse problems. J. Math. Pures Appl., 78 (1999), 65-98.

[47] X. Zhang, Explicit observability inequalities for the wave equation with lower order terms by means of Carleman inequalities. SIAM J. Control and Optim., 39 (2001), 812-834. 